\title{
Analysis of predicted full recovery time for venous leg ulcers treated with intermittent pneumatic compression
}

\author{
Paweł T. Dolibog', Patrycja Dolibog1, Daria Chmielewska² \\ ${ }^{1}$ Chair and Department of Medical Biophysics, Faculty of Medical Sciences, Medical University of Silesia, Katowice, Poland \\ ${ }^{2}$ Institute of Physiotherapy and Health Sciences, The Jerzy Kukuczka Academy of Physical Education, Katowice, Poland \\ Adv Dermatol Allergol 2022; XXXIX (1): 52-58 \\ DOI: https://doi.org/10.5114/ada.2020.99369
}

\begin{abstract}
Introduction: Venous leg ulcers are difficult-to-heal wounds. Reduction of venous hypertension and eradication of oedema is of key importance in the treatment of clinical symptoms of venous hypertension and venous leg ulcers. This can be done by using compression therapy in which external pressure is applied to the skin surface, giving a positive effect on the venous, lymphatic and arterial systems of macro- and microcirculation.

Aim: Analysis of full recovery predicted time for venous ulcers.

Material and methods: The purpose of our study was to rate the intermittent pneumatic compression (IPC) therapy in venous leg ulcers treatment. In the study group, the IPC therapy (pressure of $60 \mathrm{~mm} \mathrm{Hg}$ at the ankle) was used - one treatment daily for 4 weeks. The changes of the total area, circumference, maximum length and maximum width of ulcers were measured. Then, based on the collected values, we calculated healing progress (Gilman index), healing rate, predicted healing time and non-linear approximation of the treatment time needed to decrease the ulcer surface area by $50 \%$ and then we compared them the treatment times.

Results: Analysis of the results shows that a percentage change in the surface area in the treatment group was $52 \%$. There was a statistically significant difference between the ulcer surface area before and after treatment $(p<0.05)$. There was no statistically significant difference between the healing rate in individual weeks of treatment $(p>0.05)$. The percentage reduction in circumferences of the lower limb showed a statistically significant advantage in the study group comparison baseline $(p<0.05)$.

Conclusions: Treatment of venous leg ulcers with the IPC therapy is effective.
\end{abstract}

Key words: intermittent pneumatic compression therapy, venous leg ulcers, wound healing.

\section{Introduction}

Leg ulcers are difficult-to-heal wounds resulting from venous insufficiency. For many years, doctors and scientists have been trying to find effective treatments for both venous insufficiency and other venous ulcer diseases. It is a time-consuming process in which many people are involved and it is very expensive. With more or less success, after long years of hard work and sacrifice, we have managed to develop a new, better and more effective method of treatment.

The purpose of the healing process and the condition for the effective healing of leg ulcers is the reduction of venous pressure. In cases where surgical treatment does not produce any healing effects or cannot be performed, especially in the elderly, often burdened with additional diseases, conservative treatment is carried out.
Complementary methods include compression therapy. The standard in the treatment of patients suffering from venous leg ulcers is compression therapy. Other physical methods used to treat or support the ulceration of the leg include: high voltage electrotherapy, laser therapy, ultrasound therapy, shock wave therapy and others [1-4].

Compression increases the interstitial pressure and reduces the diameter of the veins, which leads to an increase in the speed of blood and lymph and provides the proper direction of blood flow (intracoronary). There is a reduction in pathological reflux in venous vessels and a reduction in hydrostatic pressure. The end result is a reduction in filtration pressure along with a smaller loss of fluid from the capillaries and increased resorption of the lymphatic fluid (oedema disappearance). In order to obtain compression, non-elastic bandages (Unna, Tubulcus), adjustable compression bands (CiraAid brace), elastic knee

Address for correspondence: Patrycja Dolibog PhD, Assoc. Prof., Chair and Department of Medical Biophysics, Faculty of Medical Sciences, Medical University of Silesia, 18 Medyków St, 40-752 Katowice, Poland, phone: +48 602762 442, e-mail: pdolibog@sum.edu.pl Received: 17.07 .2020 , accepted: 1.09.2020. 
socks or compression stockings, flexible bandages, multilayer compression systems (passive compression therapy) and devices producing dynamic compression are most often used (active compression therapy). For this purpose, an electronically controlled pump and cuff (sleeve) are used in which the limb is placed. Devices of this type are characterized by a wide range of pressure force regulation and duration as well as the operating mode. In addition, the cuffs are made of several or a dozen chambers, alternating with air. This provides sequential, diverse and intermittent pressure $[1-3,5]$.

The sequential intermittent pneumatic compression (active compression therapy) has been used to treat chronic venous insufficiency for several years. Onechamber compression therapy is used less frequently because it causes the closing of proximal vein lumen with the movement of the wave reducing the vessel diameter in the distal direction, while sequential compression therapy pushes the blood in the proximal direction. Our own research shows that the use of compression therapy, both active and passive, has a beneficial effect on the healing of leg ulcers in particular stages of healing and the use of active compression therapy affects the condition of the lower limb blood supply only in superficial vessels and also faster ulcer healing in patients using active compression therapy compared to passive compression therapies $[1-3,5]$.

Unfortunately, very often these new methods of treatment after many years of tests and studies turn out to be technologically outdated and everything should be restarted. And here a question arises: can you predict the treatment time before the ulcer is healed? The methods of statistical and mathematical analysis come here with help. Of course, it will not do without a clinical trial.

Using the formulas contained in Gilman and also developed by our team, we want to show that it is possible to calculate the average anticipated time of curing venous leg ulcers [6].

\section{Aim}

The aim of our study was analysis of full recovery predicted time for venous leg ulcers. For this, a linear and non-linear mathematical model was used. In addition, a thermographic analysis of wounds was performed.

\section{Material and methods}

\section{Settings and participants}

The study was conducted on 18 patients with venous leg ulcers (they had chronic venous insufficiency (CVI) like swelling, skin discoloration and lipodermatosclerosis) who were treated in the Department of Dermatology of the Medical University of Silesia, Katowice, Poland. All clinical investigations were conducted according to the principles expressed in the Declaration of Helsinki and all patients signed written consent to participate in the study. Inclusion criteria were: informed participation, active venous leg ulcer for at least 12 weeks, ankle brachial pressure index (ABPI) higher than 1, and age over 18 years. Patients with diabetes, atherosclerosis, rheumatoid arthritis, cancers, peripheral nerve damage, ventricular arrhythmia, cardiac pacemakers and pregnant women were excluded. Moreover patients who had infections of the skin, surgical treatment of ulcers, and those who reported the presence of implants of foreign bodies in the potential field of application could not participate in the study.

\section{Intervention}

In the study group of 18 patients, all the patients completed the compression therapy. Patients were treated with the intermittent pneumatic compression therapy with 12-chamber Flowtron Hydroven 12 System device (Huntleigh Healthcare, UK). Treatment parameters were as follows: pressure of $60 \mathrm{~mm} \mathrm{Hg}$ at the ankle (in higher chambers, the pressure applied was smaller and decreased with height up to $60 \%$ of the set point - Figure 1), the filling time chamber was $60 \mathrm{~s}$ and the emptying time was $30 \mathrm{~s}$, and 60-min duration of a single treatment. The therapy was provided once daily, 7 times a week for 4 weeks. After each treatment, the cuff was disinfected (Incidin Liquid Spray, Ecolab, Germany). Between treatments wet gauze dressing with saline and gently compressing elastic bandages were used. In all patients, pharmacotherapy was also used, namely micronized purified flavonoid fraction, $450 \mathrm{mg}$ diosmin, $50 \mathrm{mg}$ hesperidin once daily.

\section{Measurements}

The healing progress rates (total ulcer surface area $\mathrm{cm}^{2}$, length and width $-\mathrm{cm}$ ) were assessed using digitizer (Altek Kurta XGT, USA) and periodically wounds were photographed. The ulcer area reduction and the healing rate were evaluated in this study using Gilman index. The Gilman index is the length of the wound margin that has

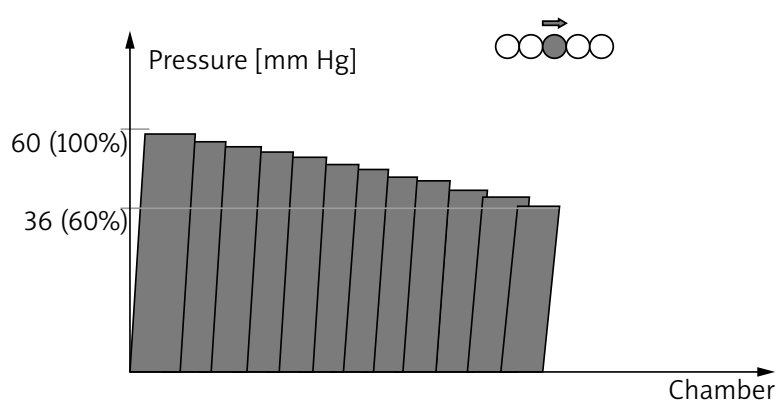

Figure 1. Treatment parameters were: pressure of $60 \mathrm{~mm} \mathrm{Hg}$ at the ankle. In higher chambers, the pressure applied was smaller and decreased with height up to $60 \%$ of the setpoint 
moved close to the centre of the wound in between the treatments. The healing rate was measured at 1-week intervals.

The Gilman index $d$ which has been designated for an exact evaluation of the healing process, was calculated using equation 1 :

$d=\frac{2\left(S_{F}-S_{1}\right)}{C_{F}+C_{1}}[\mathrm{~cm}]$

where: $S_{1}, S_{F}-$ initial and final ulcer surface area $\left(\mathrm{cm}^{2}\right) ; C_{1}$, $C_{F}$ - initial and final ulcer circumference $(\mathrm{cm})$.

According to Gilman, clinical results for venous ulcers indicate that the predicted healing time that uses the linear parameter, is calculated using equation 2 :

$H T p=\frac{\left(\operatorname{Rmax} \times{ }^{*} t\right)}{d}[$ weeks],

where: $H T_{p}$ - the predicted total time to healing; $R_{\max }-$ the radius of the largest circle that will fit entirely within the wound $(\mathrm{cm})$, or the longest distance that a wound margin will need to travel before meeting the opposite advancing wound margin $(\mathrm{cm}) ; t$ - the time it takes for the wound margin to advance distance $d$; $d$-Gilman in dex $(\mathrm{cm})$.

In our study, $d$ was calculated in first 4 weeks of treatment. Next, formula HTp was used in order to predict when the wound will be closed.

In addition, for comparison to the linear equation result, using a non-linear approximation, it was estimated when the ulcer surface area value would decrease by $50 \%$ compared to the initial value. In order to obtain homogeneity in the initial values of the ulcer area, the initial value of the reduced area for each patient was calculated using formula 3:

$S_{r e l}(t)=\frac{S(t)}{S(t=0)}\left[\mathrm{cm}^{2}\right]$

where: $S_{\text {rel }}(t)$ - relative value of the wound surface area at each week of treatment in $\mathrm{cm}^{2} ; S(t)$ - value of the wound surface area at each week of treatment in $\mathrm{cm}^{2}$ (e.g. $t=$ $0,1,2,3) ; t$ - week of treatment; $S(t=0)$ - value of the wound surface area at the start of treatment in $\mathrm{cm}^{2}$.

It follows that the initial value of the surface area reduced for each patient is 1 .

In addition, a non-linear function was used to estimate the time required to reduce the ulcer area by half in relation to the value of the ulcer (equation 4):

$S_{\text {rel }}(t)=2^{\frac{-t}{T_{1 / 2}}}\left[\mathrm{~cm}^{2}\right]$,

where: $S_{r e l}(t)$ - relative value of the wound surface area at each week of treatment in $\mathrm{cm}^{2}$; $t$ - week of treatment; $T_{1 / 2}$ - approximwate time in which the wound surface area would should decrease by half in relation to the beginning of treatment.

All patients in the study underwent ankle brachial pressure index (ABPI) examination performed using HI DOP-300 CW Pocket Doppler with Spectral Analysis device (Medizintechnik Basler AG, Switzerland) with a probe of $8 \mathrm{MHz}$.

The MobIR M3 infrared camera (Wuhan Guide Infrared Technology Co., Ltd., China) with a focal plane array uncooled microbolometer detector UFPA and a thermal sensitivity of $<120 \mathrm{mK}$ and spectral range of $8-14 \mu \mathrm{m}$ was used in this study.

The pictures were taken at a distance of $100 \mathrm{~cm}$ from the ulcer. During measurements the room temperature was $21^{\circ} \mathrm{C}$ and the humidity was $60 \%$. The images were taken at a $90^{\circ}$ angle to the camera's main axis. Before imaging, the patients underwent a 20-minute adaptation to minimize the effects of dressing temperature and clothes. The average temperatures were measured immediately before the first and last compression therapy in the wound ulcer and around the wound ulcer. The percentage of error for assessments was $\pm 2 \%$.

The thermal imaging technique enables imaging of a thermal body map, in the infrared range generated by each body heated to a temperature higher than $0 \mathrm{~K}$. This allows to use diagnostics in peripheral blood disorders and to track wound healing.

Each image was stored in the infrared camera and next the infrared camera was connected to a computer by a USB interface and all images were stored on the computer for image analysis. All images were digitally processed using Guide IrAnalyser software (Wuhan Guide Infrared Technology Co., Ltd., China, version 1.6); the average and maximal temperatures measured immediately before the first and last treatment were analysed. Each image has been filtered in the form of a contour filter. Filtration is the removal of noise from the image, and noise is the deviation of the signal from its actual value. From a mathematical point of view, the filter is a certain operator that converts one image to another using the point-by-point method. The conversion of one point into the other usually takes place taking into account the environment of the analysed point. This means that in order to determine the grayscale value or colour of a single point of the resulting image in the source image, the surrounding points need to be considered. Thanks to the contour filter used to analyse the thermographic image, it was possible to transform the source image into a mosaic and a picture with unified colours was obtained.

The percentage change of the total ulcer area, temperatures of venous leg ulcers, circumferences of the lower limb were calculated as follows:

$\Delta X \%=\frac{\left(X_{1}-X_{F}\right) \times 100 \%}{X_{1}}$,

where: $X_{1}, X_{F}$-the initial and final ulcer area $\left(\mathrm{cm}^{2}\right)$ or the initial and final temperature of venous leg ulcers $\left({ }^{\circ} \mathrm{C}\right)$ or the initial and final circumference of the lower limb $(\mathrm{cm})$. 


\section{Statistical analysis}

Statistical analyses were performed using the Statistica software 13.0 (StatSoft, Inc., version 13, USA). The values of the measured parameters were compared in the group using the non-parametric Wilcoxon test for paired observations, ANOVA Friedman test and post-hoc ANOVA Friedman rank test. The two-sided significance level of $p<0.05$ was considered statistically significant.

\section{Results}

Eighteen patients were included in the final analysis, including 6 men and 12 women, with ages ranging from 55 to 76 years, including 11 obese patients and 5 smokers. The ankle brachial pressure index (ABPI) was measured, which for all patients was higher than 0.9 (Table 1).

The average area of the ulcer before treatment in patients was $22.35 \pm 30.63 \mathrm{~cm}^{2}$ and after the end of treatment it was $12.88 \pm 17.36 \mathrm{~cm}^{2}(p=0.0007)$.

Analysis of the study group showed that initial temperatures decreased from $34.7^{\circ} \mathrm{C}$ to $34.3^{\circ} \mathrm{C}(p=0.575)$ inside the wound and decreased from $35.9^{\circ} \mathrm{C}$ to $35.4^{\circ} \mathrm{C}$ ( $p=0.333$ ) around the wound. After the end of the procedure, the wound temperature was not measured because the thermal effect caused by the cuff would distort the measurement result.

In the study we observed oedema decrease in patients who underwent compression. The percentage reduction in circumferences of the lower limb showed a statistically significant advantage in the study group comparison baseline. The comparison of the percentage reduction in oedema is shown in Table 2.

Analysis of changes in the Gilman index (equation 1) in each week of treatment showed that there were no

Table 1. Characteristics of patients

\begin{tabular}{lc}
\hline Variable & Results \\
\hline Ulcer $(n)$ & 18 \\
\hline Gender - female/male $(n)$ & $12 / 6$ \\
\hline Age [years] mean \pm SD & $66.4 \pm 7.23$ \\
\hline Height $[\mathrm{cm}]$ mean \pm SD & $166.2 \pm 9.15$ \\
\hline Weight $[\mathrm{kg}]$ mean \pm SD & $86.6 \pm 11.81$ \\
\hline Obesity $(\mathrm{BMI}) n<30 / n \geq 30$ & $7 / 11$ \\
\hline Smokers $(n)$ yes/no & $5 / 13$ \\
\hline Duration of disorder [weeks] mean \pm SD & $31.1 \pm 43.7$ \\
\hline
\end{tabular}

Table 2. Comparison of the change in the average wound surface area and circumferences of the lower limb in the study group

\begin{tabular}{|c|c|c|c|}
\hline Variable & Before therapy & After therapy & $P$-value \\
\hline \multicolumn{4}{|c|}{ Total ulcer surface area $\left[\mathrm{cm}^{2}\right]$ : } \\
\hline Mean (SD) & $22.35(30.63)$ & $12.88(17.36)$ & \\
\hline Median (min.-max.) & $5.37(0.25-113.09)$ & $2.27(0-56.58)$ & 0.0007 \\
\hline \multicolumn{4}{|c|}{ Temperature $\left({ }^{\circ} \mathrm{C}\right)$ in venous ulcers: } \\
\hline Mean (SD) & $34.7(1.68)$ & $34.3(1.4)$ & \\
\hline Median (min.-max.) & $34.1(32.6-37.7)$ & $33.9(32.2-36.3)$ & 0.575 \\
\hline \multicolumn{4}{|c|}{ Temperature $\left({ }^{\circ} \mathrm{C}\right)$ around venous ulcers: } \\
\hline Mean (SD) & $35.9(1.14)$ & $35.4(1.15)$ & \\
\hline Median (min.-max.) & $35.8(34.2-37.8)$ & $35.0(33.9-36.8)$ & 0.333 \\
\hline \multicolumn{4}{|c|}{ The circumference of the lower limb measured above the medial ankle [cm]: } \\
\hline Mean (SD) & $26.4(1.72)$ & $25.1(1.83)$ & \\
\hline Median (min.-max.) & $26.0(22.5-30)$ & $25(21.5-29)$ & 0.003 \\
\hline \multicolumn{4}{|c|}{ The circumference of the lower limb measured at the widest place of the muscular calf muscle [cm]: } \\
\hline Mean (SD) & $40.3(2.88)$ & $38.8(2.3)$ & \\
\hline Median (min.-max.) & $39.5(35-45)$ & $38.5(35-44)$ & 0.009 \\
\hline \multicolumn{4}{|c|}{ The circumference of the lower limb measured $3 \mathrm{~cm}$ below the knee joint $[\mathrm{cm}]:$} \\
\hline Mean (SD) & $39.8(2.75)$ & $38.1(2.82)$ & \\
\hline Median (min.-max.) & $39.0(34-47)$ & $38.5(34-45)$ & 0.001 \\
\hline \multicolumn{4}{|c|}{ The circumference of the lower limb measured $3 \mathrm{~cm}$ above the knee joint $[\mathrm{cm}]:$} \\
\hline Mean (SD) & $46.7(5.53)$ & $45.4(4.62)$ & 0.004 \\
\hline Median (min.-max.) & $46.0(40-64)$ & $45.0(39-57)$ & \\
\hline
\end{tabular}


Table 3. Comparison of the change in the average Gilman index in the study group

\begin{tabular}{|c|c|c|c|}
\hline \multicolumn{4}{|c|}{ Gilman index [cm] for every week of wound healing } \\
\hline $\begin{array}{l}\text { After } 1^{\text {st }} \text { week } \\
(\text { Mean } \pm \text { SD) }\end{array}$ & $\begin{array}{c}\text { After } 2^{\text {nd }} \text { week } \\
(\text { Mean } \pm \text { SD) }\end{array}$ & $\begin{array}{l}\text { After } 3^{\text {rd }} \text { week } \\
(\text { Mean } \pm \text { SD) }\end{array}$ & $\begin{array}{c}\text { After } 4^{\text {th }} \text { week } \\
(\text { Mean } \pm \text { SD) }\end{array}$ \\
\hline $0.13 \pm 0.199$ & $0.14 \pm 0.135$ & $0.07 \pm 0.129$ & $0.11 \pm 0.207$ \\
\hline
\end{tabular}

The time essential for wound area to decrease by half from baseline start treatment by 50\%: 25 days ( $95 \%$ confidence interval $0.03-0.05$ ). The vertical bars represent confidence intervals 0.95

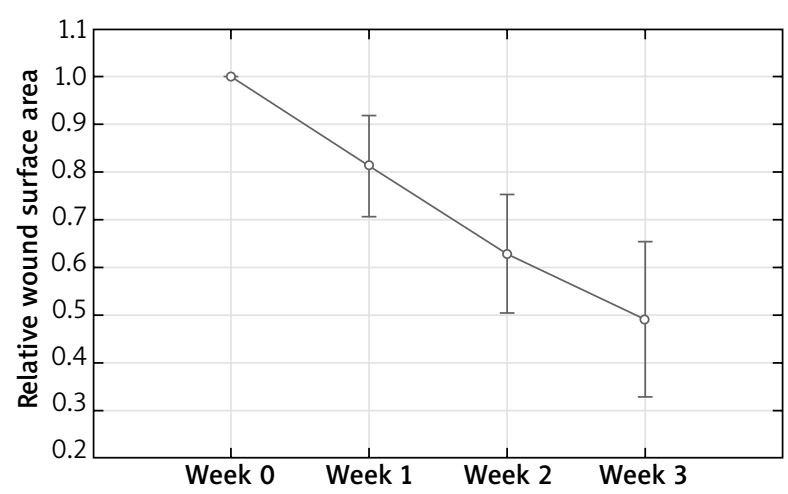

Figure 2. The nonlinear approximation of time necessary total wound surface area to decrease by half from the beginning of treatment

statistically significant differences between the healing rates in individual weeks of treatment (Table 3 ).

In this study we used equation 2 (HTp) to calculate predicted time when the wound would heal. The calculated length of treatment was 56 days. Because this is a linear equation, we can divide the result by $2-H T p / 2$ and the obtained result was 28 days (the predicted duration of treatment needed for the wound surface area to decrease from the initial value by $50 \%$ ). Next we used equation $4\left(T_{1 / 2}\right)$ to calculate predicted time when the wound would heal by $50 \%$. We calculated the duration of treatment needed for the wound surface area to decrease from the initial value by $50 \%\left(T_{1 / 2}\right)$ and it was 25 days (Figure 2 ).
The comparison shows that the result obtained from formula HTp/2 is 28 days, while for formula $T_{1 / 2}$ it is 25 days, so the results obtained are comparable (Table 4).

\section{Discussion}

Compression therapy is a standard treatment for patients with leg ulcers. Pneumatic active compression therapy is one of its varieties. The method of treatment used by us turned out to be effective in all patients.

In Dolibog et al. study [2] researchers compared five types of compression therapy in the treatment of leg ulcers: intermittent pneumatic compression, ulcer stocking system, multi-layer short stretch bandage, two-layer short stretch bandages and Unna boots. The study was conducted on 147 patients for 2 months. In the group of patients using active compression therapy (pressure of $60 \mathrm{~mm} \mathrm{Hg}$ at the ankle, $40 \mathrm{~mm} \mathrm{Hg}$ in the chamber at the level of the groin) there were 28 patients (19 women, 9 men), the average age was $62.24 \pm 9.34$ years, mean body weight $80.11 \pm 11.22 \mathrm{~kg}$, mean height $162.11 \pm 12.14 \mathrm{~cm}$, mean ulcer duration $30.12 \pm 48.56$ months, and mean ulcer size $25.16 \pm 31.17 \mathrm{~cm}^{2}$. After 2 months of daily use of active pneumatic compression, the ulcers area was reduced to an average of $10.13 \pm 20.88 \mathrm{~cm}^{2}$. The healing rate in the intermittent pneumatic compression group was $57.14 \%$, in the ulcer stocking system group $56.66 \%$, in the multi-layer short stretch bandage group $16.62 \%$, and in the Unna boots group 20\%. For the intermittent pneumatic compression group, Gilman index was $0.52 \mathrm{~cm}$ after 4 weeks.

Our study included 18 patients (12 women and 6 men) with a mean age of $66.4 \pm 7.23$ years, an average height of $166.2 \pm 9.15 \mathrm{~cm}$, an average body weight of $86.6 \pm 11.81 \mathrm{~kg}$ and mean ulcer duration of $31.1 \pm 43.7$ weeks. Before treatment the average ulcer area was $22.35 \pm 30.63 \mathrm{~cm}^{2}$.

Table 4. Comparison of calculated predicted duration of treatment needed for the wound surface area to decrease from the initial value by $50 \%$ obtained from formulas

Calculated predicted duration of treatment needed for the wound surface area to decrease from the initial value by $50 \%$

$H T p / 2$

in order to predict when the wound will be closed by $50 \%$ (equation 2)
$T_{1 / 2}$

in order to predict when the wound will be closed by $50 \%$ (equation 4) 
The treatment lasted for 4 weeks after which we noted reduction in ulcer surfaces to an average of $12.88 \pm 17.9$ $\mathrm{cm}^{2}$. The healing rate was $52 \%$. The method of treatment used by us is comparable to the methods of other researchers and the results of the treatment we have obtained are compatible.

In another study [3], researchers compared three types of compression therapy: intermittent pneumatic compression, stockings, and short-stretch bandages to treat 70 patients with venous ulcers. The researchers additionally divided patients to a group with isolated superficial vein insufficiency and a group with combined superficial and deep vein insufficiency. In the group of patients with superficial reflux who were using active compression therapy (12-chamber intermittent pneumatic compression therapy) the mean total ulcer surface area before therapy was $20.12 \pm 30.07 \mathrm{~cm}^{2}$. Treatments were performed once daily for 15 days; a single treatment lasted $60 \mathrm{~min}$. The average total ulcer surface area was $10.21 \pm 21.5 \mathrm{~cm}^{2}$. In the group of patients with combined superficial and deep vein insufficiency who received active compression therapy, the mean total ulcer surface area before therapy was $19.35 \pm 10.89 \mathrm{~cm}^{2}$. Treatments were performed once daily for 15 days; a single treatment lasted $60 \mathrm{~min}$. The average total ulcer surface area after therapy was $10.02 \pm 21.32 \mathrm{~cm}^{2}$. The healing rate is $41.22 \%$ for isolated superficial vein insufficiency and $38.68 \%$ for combined superficial and deep vein insufficiency. The authors conclude that intermittent pneumatic compression therapy showed a much faster reduction in wound surface than the ulcers treated with two-layer, short-stretch bandages and standard care. This applies to both combined superficial and deep vein insufficiency or isolated superficial alone. The Gilman index was not determined by the authors.

Our treatment results concern the longer end of treatment, therefore the healing rate in the cases described above is slightly lower.

Alvarez et al. [7] treated 52 patients with venous ulcers (25 - intermittent pneumatic compression plus standard compression therapy and 27 - standard compression therapy alone). Intermittent pneumatic compression was performed using a 4-chamber pneumatic leg sleeve (50 $\mathrm{mm} \mathrm{Hg}$ ); treatments were performed for $1 \mathrm{~h}$, twice daily. The authors gave a median of the total healing time. For patients treated for 9 months using pneumatic compression, the median was 141 days and 211 days for the control group.

The difference in the length of the total healing time in relation to our results may be due to the fact that Alvarez et al. treated venous leg ulcers with a very large surface above $20 \mathrm{~cm}^{2}$ and patients with secondary lymphedema.

Harding et al. [8] have treated patients with leg ulcers for 12 weeks using an adaptive compression therapy system, combining intermittent and sustained pneumatic compression ( $n=38$ ) and a conventional four-layer bandage system $(n=52)$. Active compression therapy was applied every day during $71 \%$ of waking hours (sustained compression of $40 \mathrm{~mm} \mathrm{Hg}$, intermittent compression of $50 \mathrm{~mm} \mathrm{Hg}$ ). Complete recovery was reported in 32\% of patients treated with active compression and in $42 \%$ of patients treated with bandages. Authors, using the Kaplan-Meier analysis, calculated time to healing as 64 days for patients from the group of active compression therapy and 48.5 days for patients from the bandage group.

The results of our analysis indicate a slightly different time of treatment duration, however, differences in the duration of daily treatments and the surface of ulcers taken for treatment by Harding et al. (less than $15 \mathrm{~cm}$ in length) can be the cause.

The results obtained in our study do not differ significantly from those obtained by other authors.

Dissemond et al. [9] suggest that various compression therapies should be used to treat ulcers depending on the healing phase. In the initial phase of the treatment (maintenance phase) the compression therapy based on the system of compression knee socks is used and in the subsequent phase (decongestion phase) compression bandages should be used. However, the use of bandages or other forms of compression depending on human abilities requires correct application, good training and monitoring, which is often lacking in daily practice. Hence the researchers suggest using in the second phase of the healing, intermittent sequential compression therapy which allows for constant pressure during therapy, what is extremely difficult to achieve with compressions bandage.

Mayrovitz et al. [10] conducted a study of the influence of the adaptive compression therapy device and a traditional 4-layer bandage system among 12 healthy volunteers. Immediately after the procedure and after 1 , 4 and $8 \mathrm{~h}$, the pressure in the sitting and standing positions in the lower, middle and upper calves was measured. Both in sitting and standing positions immediately after the procedure, pressure in all measured points was greater for a group of volunteers using a bandage. After 1 , 4 and $8 \mathrm{~h}$, the researchers noted a drop in blood pressure relative to the initial pressure at all points measured in volunteers using bandages (in both sitting and standing positions). In contrast, volunteers using adaptive compression therapy experienced a significant increase in pressure at all measured points after 1,4 and $8 \mathrm{~h}$ (in both sitting and standing positions).

Another study of Dolibog et al. [11] used calculations of the nonlinear approximation of treatment results $\left(T_{1 / 2}\right)$ which for radial shockwave therapy (R-ESWT) was 7.8 weeks (54 days) and focused shockwave therapy (F-ESWT) 5.5 weeks (39 days). 
In our study, the time needed to decrease the wound surface area by $50 \%$, which is twice as less than in the radial shockwave therapy group.

Compression therapy is a proper and effective method supporting the treatment of leg ulcers. The large variety of methods makes it possible to choose the therapy individually for the patient. In addition, it is possible to combine several compression methods, depending on the healing phase or other factors.

It is significant that the medical method be effective and the effect of treatment maintain longer. The effectiveness of the treatment method is also the duration of therapy after which we can achieve the desired goal: healed wounds. The possibility of planning treatment in the context of the duration of treatment is important from the point of view of the physician planning treatment and the patient who often had used other types of treatment before.

Mathematical analysis of the planned healing time also allows you to monitor the wound healing time with a given method, and if this time increases considerably, other more effective methods should be used or causes of slower wound healing should be established. Statistical and mathematical analysis is necessary in the planning and monitoring of wound healing.

The limitation of our study is the small number of patients and the relatively short period of observation of patients. In addition, it would be necessary to compare the calculation results with the measurement results after the time calculated by us as the anticipated recovery time.

\section{Conclusions}

Intermittent pneumatic compression therapy (pressure of $60 \mathrm{~mm} \mathrm{Hg}$ at the ankle) given as one treatment daily for 4 weeks is an effective method of treating venous leg ulcers.

Statistical and mathematical analysis based on the data collected by us shows that for active compression therapy, the mean total healing time for ulcers is from 78 to 84 days from the day the treatment started.

\section{Conflict of interest}

The authors declare no conflict of interest.

\section{References}

1. Taradaj J, Franek A, Cierpka L, et al. Early and long-term results of physical methods in the treatment of venous leg ulcers: randomized controlled trial. Phlebology 2011; 26: 237-45.

2. Dolibog P, Franek A, Taradaj J, et al. A comparative clinical study on five types of compression therapy in patients with venous leg ulcers. Int I Med Sci 2014; 11: 34-43.

3. Dolibog P, Franek A, Taradaj J, et al. A randomized, controlled clinical pilot study comparing three types of compression therapy to treat venous leg ulcers in patients with superficial and/or segmental deep venous reflux. Ostomy Wound Manage 2013; 59: 22-30.

4. Collins L, Seraj S. Diagnosis and treatment of venous ulcers. Am Fam Physician 2010; 15: 989-96.

5. Brijesh N. Compression therapy for venous leg ulcers. Indian Dermatol Online J 2014; 5: 378-82.

6. Gilman T. Wound outcomes: the utility of surface measures. Int J Low Extrem Wounds 2004; 3: 125-32.

7. Alvarez O, Wendelken M, Markowitz L, et al. Effectiveness of intermittent pneumatic compression for the treatment of venous ulcers in subjects with secondary (acquired) lymphedema. Vein 2012; 5: 32-4.

8. Harding KG, Vanscheidt W, Partsch $\mathrm{H}$, et al. Adaptive compression therapy for venous leg ulcers: a clinically effective, patient-centredapproach. Int Wound J 2016; 13: 317-25.

9. Dissemond J, Eder S, Läuchli S, et al. Kompressionstherapie des Ulcus cruris venosum in der Phase der Entstauung. Med Klin Intensivmed Notfmed 2018; 113: 552-9.

10. Mayrovitz HN, Partsch H, Vanscheidt W. Comparison of 4-layer bandages and an adaptive compression therapy device on intended pressure delivery. I Wound Ostomy Continence Nurs 2015; 42: 468-73.

11. Dolibog P, Dolibog P, Franek A, et al. Randomized, controlled clinical pilot study of venous leg ulcers treated with using two types of shockwave therapy. Int I Med Sci 2018; 15: 1275-85. 